Mrtchison, D. A. (1953). J. gen. Microbiol. 8, 168-185.

\title{
The Occurrence of Independent Mutations to Different Types of Streptomycin Resistance in Bacterium coli
}

\author{
By D. A. MITCHISON \\ Department of Bacteriology, Postgraduate Medical School of London, \\ Ducane Road, London, W.12
}

SUMMARY: Streptomycin resistant strains of Bacterium coli were obtained by plating numerous 1 or $1.5 \mathrm{ml}$. volume cultures of a sensitive strain on plates containing $100 \mu \mathrm{g}$. streptomycin $/ \mathrm{ml}$. The growth rate of these strains was measured by mixing them with the sensitive strain and measuring the fall in the numbers of resistant organisms during several periods of daily subculture in streptomycin free broth. Their resistance to streptomycin and stability during successive subcultures in broth were also studied. They could be divided into three main types. All type I strains had identical slow growth rates, were completely resistant, though growing poorly at high streptomycin concentrations and were quickly replaced by rapidly growing slightly resistant variants when subcultured in broth. All type II strains had identical slightly more rapid growth rates, were partially resistant and gradually decreased their resistance in a stepwise manner during subculture. Type III strains had growth rates slightly less than that of the sensitive strain and of a degree of variability similar to those found in clones of the sensitive strain. They were completely resistant and were stable during subculture. They could be subdivided on the basis of one characteristic. Type III $a$ strains grew as well in the presence or absence of streptomycin but type III $b$ strains grew much more slowly in the presence of $1600 \mu \mathrm{g}$. ml. streptomycin or over. Since all the strains from any one plate were usually of the same type they were considered to have arisen from a single mutant cell. Since each mutation occurred several times in independent cultures and the number of tubes in which multiple mutations occurred was not greater than would be expected by chance, each type is considered to have arisen by an independent mutation.

Bacterial populations sensitive to streptomycin have been shown to contain small numbers of resistant organisms. Using Bacterium coli strain $\mathrm{B} / \mathrm{r}$, Newcombe \& Hawirko (1949) studied the number of organisms growing in plates containing graded concentrations of streptomycin and found two types of resistant strains. One of these was 2-4 times less sensitive than the parent strain, which was inhibited by about $8 \mu \mathrm{g} . / \mathrm{ml}$. The other type, obtained from plates containing concentrations of streptomycin above $16 \mu \mathrm{g} . / \mathrm{ml}$., was capable of growth in concentrations of at least $1000 \mu \mathrm{g} . / \mathrm{ml}$. Meads \& Haslam (1949) obtained similar results with Bact. friedlaenderi. Alexander \& Leidy (1947) found no difference between the number of colonies of Haemophilus influenzae which appeared when a given suspension was seeded in media in the presence of 100 and $1000 \mu \mathrm{g} . / \mathrm{ml}$.

In the course of experiments with streptomycin resistant strains of Bact. coli obtained by plating out a sensitive strain in the presence of $100 \mu \mathrm{g} . / \mathrm{ml}$. it became clear that resistant strains could be divided into a number of types. The purpose of this paper is to show that in such strains four distinct types of 
resistant organisms may be recognized in addition to dependent organisms, and that each arises by independent mutations. The strains have been studied from the point of view of growth rate, degree and type of resistance to streptomycin, and stability during successive cultures in medium free of streptomycin. In addition, it will be shown that strains adapted to streptomycin by multiple steps differ from any of these types, though most closely resembling one of them.

\section{METHODS}

Strain of Bacterium coli. A typical strain of Bact. coli var. communis was used, inhibited by a streptomycin concentration of about $5 \mu \mathrm{g} . / \mathrm{ml}$. A Dorset's egg slope was inoculated from a single colony and, after incubation, kept in the refrigerator. An $18 \mathrm{hr}$. broth culture inoculated from this was used for the inoculum in all the experiments.

Medium. The medium used was a modified Hartley's digest broth containing tryptic digest of beef and adjusted to $\mathrm{pH} 7 \cdot 5$. Solid media contained $1.5 \%$ $(\mathrm{w} / \mathrm{v})$ agar.

Procedure. A series of tubes containing $1 \mathrm{ml}$. of broth was inoculated with one loopful of an $18 \mathrm{hr}$. broth culture diluted so that each loopful contained 50-200 organisms. After incubation at $37^{\circ}$ for 18-24 hr. the contents of each tube were spread over the surface of an overdried nutrient agar plate containing $100 \mu \mathrm{g}$. streptomycin $/ \mathrm{ml}$., a few of the tubes being reserved for a viable count. The plates were incubated for $\mathbf{4 8} \mathrm{hr}$. Any colonies which grew were counted and each colony was subcultured $(a)$ on to an agar slope or a Dorset's egg slope which was kept in the refrigerator as a stock culture, $(b)$ on to a portion of a streptomycin ditch plate in order to distinguish between resistant and dependent strains, and $(c)$ into a tube of plain broth which was incubated for $18 \mathrm{hr}$. and then used for determining the growth rate. Strains derived from colonies from the same plate which had similar characteristics were said to be derived from the same resistant clone. Dependent strains were discarded.

In some experiments a $50 \mathrm{ml}$. volume of broth was also inoculated with the sensitive strain. After incubation the centrifuged deposit was divided between a number of streptomycin plates, and the colonies from one of these were investigated in a similar manner.

Viable counting. A dropping pipette calibrated by weighing to deliver $0.02 \mathrm{ml} . / \mathrm{drop}$ and a single $3 \mathrm{~mm}$. loop calibrated by weighing to remove $0.0044 \mathrm{ml}$./loopful were used. With a pipette $0.5 \mathrm{ml}$. of culture was added to $9.5 \mathrm{ml}$. of diluent ( $1 \%$ broth in distilled water). From this two $0.02 \mathrm{ml}$. drops were added to bottles containing 0.96, 10 and $100 \mathrm{ml}$. diluent. From the appropriate dilutions a loopful was spread over a quarter of the surface of a plate. With rapidly growing strains the plates were incubated for $18 \mathrm{hr}$. at $30^{\circ}$, whereas, with slowly growing strains, they were incubated at $37^{\circ}$. In this way 200-300 colonies could easily be counted on each segment of a plate. A fully grown broth culture usually yielded 100 colonies from the highest dilution. In a series of duplicate counts from such cultures the coefficient of variation was $c .11 \%$, the theoretical figure from the number of colonies 
counted being $9 \%$. The pipette and the calibrated dropper were sterilized between counts by pipetting boiling water in and out several times. Using this method a count could be done rapidly and with few materials.

Growth rates. An indirect method, which has not been previously described, was used for determining growth rates. Equal volumes of the first broth subculture from a resistant colony and of a similar broth culture of the sensitive strain were mixed. From this mixture a viable count was done on plates with

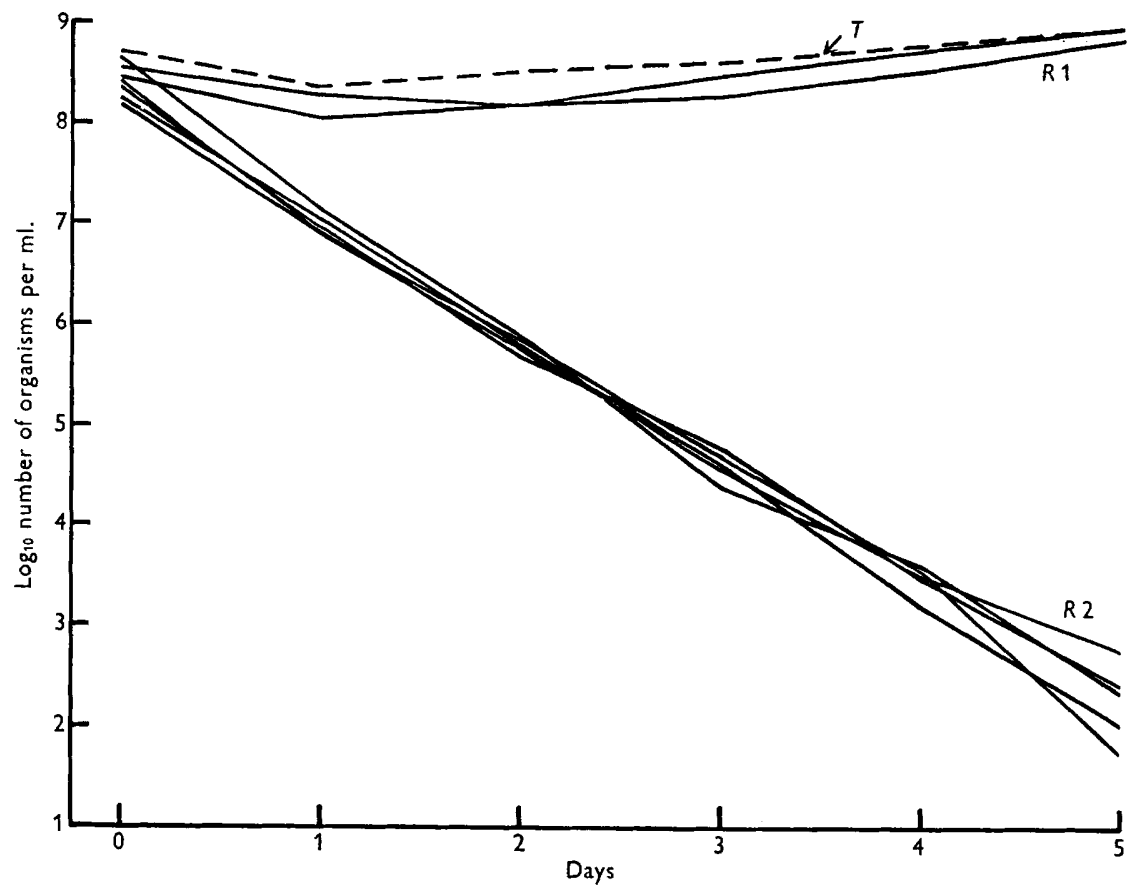

Fig. 1. $T$, total count. $R 1$, group of two resistant strains growing at the same rate as the sensitive strain. $R 2$, group of nine resistant strains of which only five are represented for the sake of clarity, growing at a slower rate than the sensitive strain.

and without streptomycin $100 \mu \mathrm{g} . / \mathrm{ml}$., so that the total number of organisms and the number of resistant organisms was known. A standard $3 \mathrm{~mm}$. loopful $(0.005 \mathrm{ml}$.) of the mixture was also subcultured into a tube of broth. After $\mathbf{2 4} \mathrm{hr}$. incubation a differential viable count was made from the second tube and the mixed growth was subcultured with the same loop into a fresh tube of broth. This procedure was repeated at daily intervals. In most of the experiments counts were done on the initial mixture and on the mixture after either one and two or two and four daily subcultures according to the rapidity with which the resistant strain was eliminated. The same batch of broth was used throughout any one experiment.

In Fig. 1 an experiment illustrating the method is shown in which two clones of resistant strains having growth rates the same and much slower than the sensitive strain were tested simultaneously. For any of these strains there 
was a straight-line relationship between the number of resistant organisms found in the mixture and the number of successive daily subcultures. The linearity of the relationship was proved in this and other experiments by an analysis of variance. The slope of this line, $\mathrm{g}_{I}$, was used as a measure of the differential growth rate.

The relationship between $g_{I}$ and the increase in numbers of the resistant and sensitive strains, during their logarithmic phases of growth, $g_{r}$ and $g_{s}$, can be seen from consideration of Fig. 2.

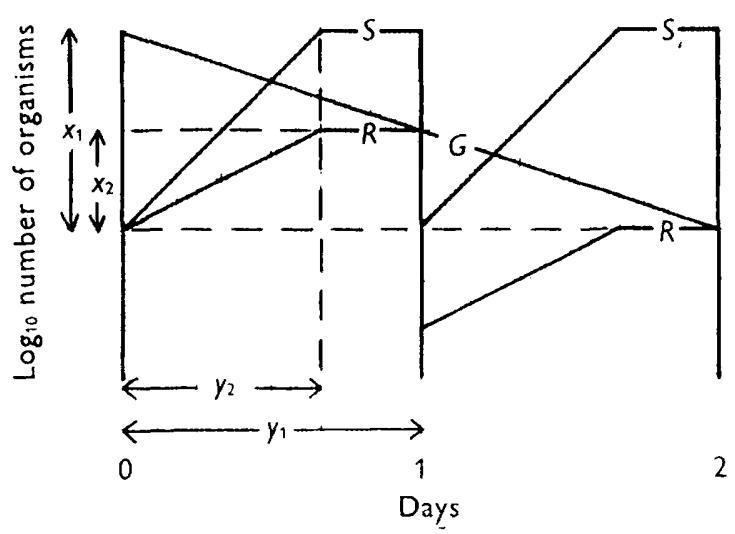

Fig. 2. Diagrammatic representation of the growth of a mixture of a sensitive and a resistant strain during two successive subcultures. $S$, growth curve of sensitive strain; $R$, growth curve of resistant strain; $G$, line representing the fall in the numbers of the resistant strain; $x_{1}$, the increase in concentration of the sensitive strain during a $24 \mathrm{hr}$. period of culture; $x_{2}$, the increase in concentration of the resistant strain during the same period; $y_{1}$, the duration of a period of culture $=1$ day; $y_{2}$, the period during which growth takes place.

Let $g_{s}=$ growth rate of the sensitive strain; $g_{r}=$ growth rate of the resistant strain; $g_{I}=$ slope of the line relating fall in number of resistant organisms to period of subculture in days. This line corresponds to those illustrated in Fig. 1. Then

$$
g_{s}=\frac{x_{1}}{y_{2}}, \quad g_{r}=\frac{x_{2}}{y_{2}}, \quad g_{I}=-\frac{x_{1}-x_{2}}{y_{1}},
$$

$x_{2}$ and $y_{2}$ are eliminated from these equations, and $y_{1}=1$, so that:

$$
\frac{g_{r}}{g_{s}}=\frac{x_{1}+g_{I}}{x_{1}}
$$

Assuming that the growth rate of the sensitive strain is constant, and known, the growth rate of the resistant strain can be calculated from $g_{I}$ if the logarithm of the ratio between the volume of the inoculum and the volume of the tube of broth $\left(x_{1}\right)$ is determined. This assumes that the final concentration of the organisms after each growth period is the same.

Viable counts on plates with and without streptomycin were done at hourly intervals during the growth of a mixture of the sensitive and a resistant strain. The form of these curves, shown in Fig. 3, corresponds to the diagrammatic representation in Fig. 2. 
The indirect method of measuring growth rates is particularly well suited to measure small differences in growth rates between several strains. It was found possible to investigate up to forty strains simultaneously. Any variations in the experimental conditions, such as the final concentration in the cultures, affected each strain to the same extent and did not destroy the validity of the comparison.

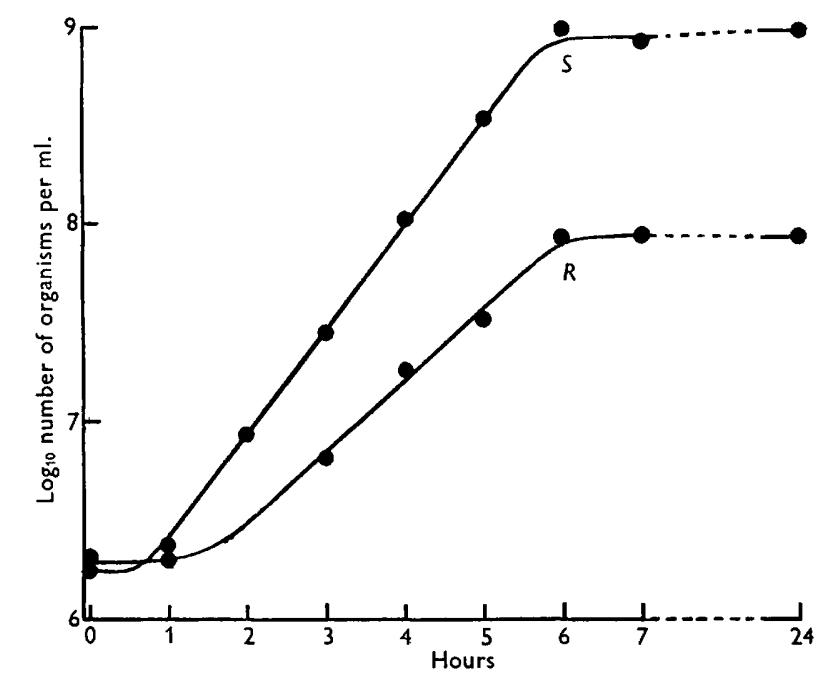

Fig. 3. Growth curves of the sensitive and a resistant strain growing together in broth. $S$, sensitive strain; $R$, resistant strain.

As a check on this method viable counts were done at hourly intervals during the logarithmic phase of growth on a series of five strains, including the sensitive strain. The indirect method was done at the same time in the same batch of broth. It was possible to compare the growth rates obtained by measuring the slopes of the logarithmic portion of the growth curves with the estimates of these growth rates obtained from the values of the differential growth rates (indirect method) by the use of equation (1). The results are given in table 1. There was no significant difference between the two estimates for each resistant strain. The indirect method yielded values of $g_{I}$ from 0 to $-\mathbf{1 \cdot 2 4}$, having standard errors of \pm 0.045 to \pm 0.090 . It was slightly more accurate than the direct method which gave values of $g_{s}$ or $g_{r}$ from 0.845 to 0.463 with standard errors of \pm 0.059 to \pm 0.032 .

Streptomycin sensitivity. Where a large number of strains was being tested simultaneously nutrient agar plates were prepared containing concentrations of streptomycin in the series $0,1,2,4, \ldots, 6400 \mu \mathrm{g} . / \mathrm{ml}$. A $1 \mathrm{~mm}$. loopful of a $24 \mathrm{hr}$. broth culture of the strain, usually derived from the slope stored in the refrigerator, was inoculated into $3 \mathrm{ml}$. of broth. A loopful of this dilution was then spread on a small area of each of the plates. It was possible to test at least twenty strains on one set of plates.

Stability of the strains. The stability during passage through streptomycin 
free broth was investigated for a few strains of each of the types described. The strains were subcultured at daily intervals in broth, using a $3 \mathrm{~mm}$. loopful as the inoculum. The streptomycin sensitivity of the strains was determined daily for the first few days, and later at approximately weekly intervals. A loopful of the highest dilution described under the method for viable counting was spread over a quarter of each of a series of plates containing a suitable range of streptomycin concentrations and the number of colonies counted. Cultures of known sensitivity were always included with each batch of strains tested in order to control the slight variations encountered with different batches of nutrient agar.

Table 1. Comparison of growth rates determined directly and by estimation from the indirect method

\begin{tabular}{|c|c|c|}
\hline \multirow[b]{2}{*}{ Strain } & \multicolumn{2}{|c|}{ Growth rate, $g_{s}$ or $g_{r}$} \\
\hline & $\begin{array}{l}\text { Estimated } \\
\text { from } g_{I}{ }^{*}\end{array}$ & $\begin{array}{l}\text { Estimated } \\
\text { directly }\end{array}$ \\
\hline $\mathbf{S}$ & 0.845 & 0.845 \\
\hline R1 & $0 \cdot 769$ & 0.830 \\
\hline R2 & 0.672 & 0.629 \\
\hline R3 & 0.639 & 0.667 \\
\hline R4 & 0.496 & 0.463 \\
\hline
\end{tabular}

* Using equation (1) in the text, $x_{1}=3 \cdot 00$.

$g_{s}$ or $g_{r}$ are the $\log _{10}$ increases in numbers of sensitive or resistant organisms per $\mathrm{hr}$. of growth in nutrient broth at $37^{\circ}$. These were obtained directly or by estimation from values of the fall in numbers of resistant organisms per day of subculture, $g_{I}$, as described in the text.

A note was made of the size and the amount of variation in the sizes of colonies on plain agar. On occasion the size of colonies was measured with a low-power microscope and a calibrated eyepiece.

Production of adapted strains. A series of tubes of broth containing a range of streptomycin concentrations was inoculated with two drops of a $24 \mathrm{hr}$. broth culture of the sensitive strain. After $24 \mathrm{hr}$. incubation another set was inoculated from the tube with the highest streptomycin concentration that showed growth. After five such transfers of two strains each showed partial growth in $1000 \mu \mathrm{g} . / \mathrm{ml}$. These strains were tested for growth rate, streptomycin sensitivity and stability as described previously.

\section{RESULTS}

\section{Growth rates}

The results of two experiments are given in Table 2. The strains derived from resistant colonies could be divided into three types. Type I strains yielded very small colonies on plain nutrient agar and were rapidly eliminated from a mixture with the sensitive strain. Type II strains yielded slightly larger colonies and were eliminated rapidly but at a slower rate than type I strains. Type III strains yielded larger colonies of variable size. They were eliminated at variable rates from the mixtures, usually less rapidly than either type $I$ or 
type II strains. They could be divided, as will be explained later, into two subtypes, III $a$ and III $b$, but this distinction was not made in Exp. 1.

It seemed clear from these two experiments that the strains derived from any one plate seeded with $1 \mathrm{ml}$. of broth culture were usually of one type and where these were of type III they had the same growth rates. Occasionally

Table 2. The classification and growth rates of resistant strains obtained by inoculating streptomycin plates with $1 \mathrm{ml}$. volumes of broth cultures of the sensitive strain

Exp. 1. Average number of organisms in each $1 \mathrm{ml}$. culture $1.69 \times 10^{9}$.

Total number of plates receiving $1 \mathrm{ml}$. culture: 37 .

A. Plates yielding resistant colonies

\begin{tabular}{|c|c|c|c|c|c|c|c|}
\hline \multirow{2}{*}{$\begin{array}{c}\text { Plate } \\
\text { no. }\end{array}$} & \multirow{2}{*}{$\begin{array}{c}\text { No. of } \\
\text { dependent } \\
\text { colonies }\end{array}$} & \multirow{2}{*}{$\begin{array}{l}\text { No. of } \\
\text { resistant } \\
\text { colonies }\end{array}$} & \multicolumn{5}{|c|}{ Classification of colonies and differential growth rates, $g$} \\
\hline & & & \multicolumn{2}{|c|}{ Type I } & Type II & \multicolumn{2}{|c|}{ Type III } \\
\hline 1 & o & 1 & \multicolumn{2}{|c|}{-} & 一 & \multicolumn{2}{|c|}{$-0 \cdot 655$} \\
\hline 2 & 0 & 1 & \multicolumn{2}{|c|}{ - } & - & \multicolumn{2}{|c|}{$-0 \cdot 565$} \\
\hline 3 & o & 6 & $\begin{array}{l}-2 \cdot 150 \\
-2 \cdot 155 \\
-2 \cdot 200\end{array}$ & $\begin{array}{l}-2 \cdot 165 \\
-2 \cdot 255 \\
-2 \cdot 240\end{array}$ & - & & \\
\hline 4 & 0 & 3 & \multicolumn{2}{|c|}{-} & $\begin{array}{l}-1.488 \\
-1.488 \\
-1.425\end{array}$ & \multicolumn{2}{|c|}{-} \\
\hline 5 & 0 & 2 & \multicolumn{2}{|c|}{ 一 } & - & -1.093 & -0.983 \\
\hline 6 & 1 & 2 & $-2 \cdot 210$ & $-2 \cdot 195$ & 一 & \multicolumn{2}{|c|}{-} \\
\hline $7^{*}$ & 0 & 21 & \multicolumn{2}{|c|}{ 一 } & - & \multicolumn{2}{|c|}{ - } \\
\hline $\begin{array}{c}8 \\
\text { (seeded } \\
\text { from part of } \\
\text { a } 50 \mathrm{ml} \text {. } \\
\text { culture) }\end{array}$ & 25 & 23 & $\begin{array}{l}-2 \cdot 195 \\
-2 \cdot 160\end{array}$ & $\begin{array}{l}-2 \cdot 195 \\
-2 \cdot 210\end{array}$ & $\begin{array}{l}-1.638 \\
-1.518 \\
-1.443\end{array}$ & $\begin{array}{l}-0.270 \\
-0.248 \\
-0.265 \\
-0.560 \\
-0.558 \\
-0.575 \\
-0.585 \\
-0.623\end{array}$ & $\begin{array}{l}-0.295 \\
-0.263 \\
-0.278 \\
-0.565 \\
-0.580 \\
-0.575 \\
-0.713 \\
-0.573\end{array}$ \\
\hline
\end{tabular}

B. Plates on which only dependent organisms grew: Number of plates: 14; numbers of dependent colonies on these plates: $2,3,4,1,5,2,2,2,1,1,1,2,1,1$.

C. Number of plates not yielding growth: 16 .

* One plate yielded twenty-one type III colonies, whose growth rates were not tested.

two types were present, but the colonies could always be distinguished from each other on the original streptomycin plates. For this reason in the third and fourth experiments detailed in Table 3, where there were numerous colonies of the same size on a streptomycin plate only a few of them were sampled. Where more than one size of colony was present on any plate samples of each size were tested. In the fourth experiment $1.5 \mathrm{ml}$. of broth culture were inoculated on to plates containing 1000 instead of $100 \mu \mathrm{g} . / \mathrm{ml}$. streptomycin and only two colonies from each plate were tested. The main purpose of this experiment was to obtain a number of separate clones of type III strains for an investigation of the variability of their growth rates. As will be noted later 
Table 2 (cont.)

Exp. 2. Average number of organisms in each $1 \mathrm{ml}$. culture $2 \cdot 28 \times 10^{9}$.

Total number of plates receiving $1 \mathrm{ml}$. culture: 29 .

A. Plates yielding resistant colonies : 11

\begin{tabular}{|c|c|c|c|c|c|c|c|}
\hline \multirow{2}{*}{$\begin{array}{c}\text { Plate } \\
\text { no. }\end{array}$} & \multirow{2}{*}{$\begin{array}{l}\text { No. of } \\
\text { dependent } \\
\text { colonies }\end{array}$} & \multirow{2}{*}{$\begin{array}{l}\text { No. of } \\
\text { resistant } \\
\text { colonies }\end{array}$} & \multicolumn{5}{|c|}{ Classification of colonies and differential growth rates, $g$} \\
\hline & & & Type I & Type II & Type & III $a$ & Type III $b$ \\
\hline 1 & 0 & 1 & - & - & -1 & 390 & - \\
\hline 2 & 2 & 2 & - & - & $-0 \cdot 733$ & -0.743 & - \\
\hline 3 & 2 & 7 & $-2 \cdot 395$ & - & $\begin{array}{l}-0.545 \\
-0.505 \\
-0.540\end{array}$ & $\begin{array}{l}-0.474 \\
-0.534 \\
-0.494\end{array}$ & 一 \\
\hline 4 & 0 & 1 & $-2 \cdot 265$ & 一 & - & & - \\
\hline 5 & 1 & 5 & $-2 \cdot 270$ & - & $\begin{array}{l}-0.617 \\
-0.533\end{array}$ & $\begin{array}{l}-0.549 \\
-0.567\end{array}$ & - \\
\hline 6 & 1 & 1 & - & $-1 \cdot 430$ & - & & - \\
\hline 7 & 0 & 2 & - & - & 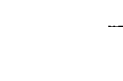 & & $\begin{array}{l}-0.377 \\
-0.303\end{array}$ \\
\hline 8 & 3 & 4 & $\begin{array}{l}-2 \cdot 165 \\
-2 \cdot 295\end{array}$ & $\begin{array}{l}-1 \cdot 240 \\
-1 \cdot 475\end{array}$ & - & & - \\
\hline 9 & 0 & 3 & $-2 \cdot 210$ & - & -0.526 & $-0 \cdot 468$ & - \\
\hline 10 & 1 & 4 & - & - & $\begin{array}{l}-0.498 \\
-0.556\end{array}$ & $\begin{array}{l}-0.572 \\
-0.654\end{array}$ & - \\
\hline 11 & 0 & 1 & - & - & -0 & 632 & - \\
\hline $\begin{array}{c}12 \\
\text { (seeded } \\
\text { from part of } \\
\text { a } 50 \mathrm{ml} . \\
\text { culture) }\end{array}$ & 8 & 17 & $-2 \cdot 195$ & - & $\begin{array}{r}-0.240 \\
-0.271 \\
-0 . \\
-0.603 \\
-0.501 \\
-0.625 \\
-0.613 \\
-0.614 \\
-0 .\end{array}$ & $\begin{array}{l}-0 \cdot 238 \\
-0 \cdot 241 \\
256 \\
-0 \cdot 537 \\
-0 \cdot 537 \\
-0 \cdot 608 \\
-0 \cdot 588 \\
-0 \cdot 646 \\
567\end{array}$ & - \\
\hline
\end{tabular}

B. Plates on which only dependent organisms grew : number of plates : 7 ; numbers of dependent colonies on these plates: $1,2,1,1,2,3,4$.

C. Number of plates not yielding growth: 11 .

type II strains were inhibited by streptomycin at $1000 \mu \mathrm{g} . / \mathrm{ml}$. so that none of them was obtained on these plates.

Values of the differential growth rates obtained in these experiments were subjected to standard analysis of variance methods. The following conclusions could be drawn:

(1) Within the limits of experimental error there was no difference in the growth rates of strains belonging to the same clone.

(2) All type I strains had identical growth rates.

(3) All type II strains had identical growth rates which were more rapid than those of type I strains.

(4) Each clone of type III strains had its own characteristic growth rate which differed from those of other clones. 
Table 3. Classification and growth rates of samples of the resistant strains obtained by inoculating streptomycin plates with $1 \mathrm{ml}$. volumes of broth cultures of the sensitive strain

Exp. 3. Average number of organisms in each $1 \mathrm{ml}$. culture $1.29 \times 10^{9}$.

Total number of plates: 41 .

A. Plates yielding resistant colonies : 8 .

No. of No. of dependent resistant

Plate no. colonies colonies

1
2
3
4

5
6
7
8

0
0
5
0
0
2
0
1

0

3

1

1

10

0

2

0

Classification of colonies and differential growth rates, $g_{I}$

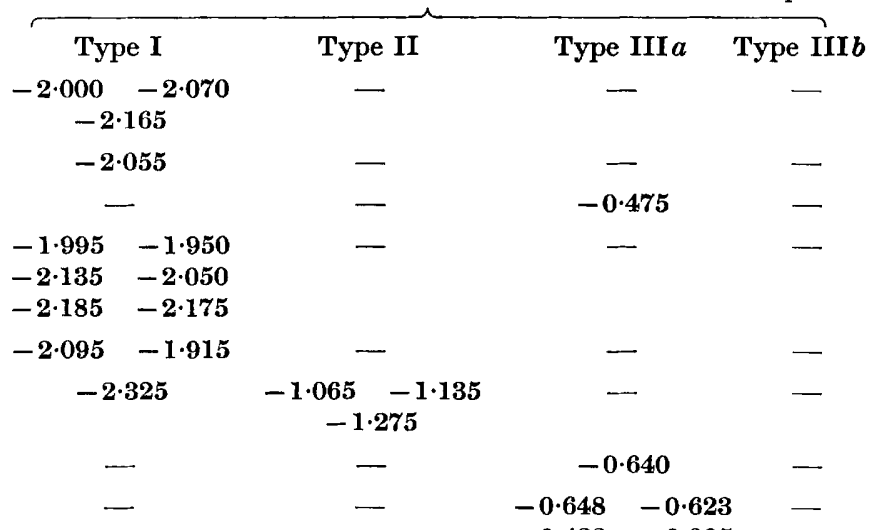

B. Plates on which dependent organisms only grew : number of plates: 10 ; numbers of dependent colonies on these plates: $2,1,4,2,1,2,2,6,1,2$.

C. Number of plates not yielding growth: 23 .

Exp. 4. Broth cultures of $1.5 \mathrm{ml}$. volume inoculated on to plates containing streptomycin $1000 \mu \mathrm{g} . / \mathrm{ml}$.

\begin{tabular}{|c|c|c|c|c|c|}
\hline \multirow{2}{*}{$\begin{array}{l}\text { Plate } \\
\text { no. }\end{array}$} & \multirow{2}{*}{$\begin{array}{l}\text { No. of } \\
\text { colonies on } \\
\text { plate }\end{array}$} & \multicolumn{4}{|c|}{$\begin{array}{c}\text { Classification of sample of two colonies from each plate and } \\
\text { differential growth rates, } g_{I}\end{array}$} \\
\hline & & Type I & Type III $a$ & \multicolumn{2}{|c|}{ Type III $b$} \\
\hline 1 & 26 & $-2 \cdot 145 \quad-2 \cdot 275$ & - & - & \\
\hline 2 & $\mathbf{5}$ & $-2 \cdot 280-2 \cdot 395$ & - & - & \\
\hline 3 & 59 & - & $-0.720 \quad-0.648$ & - & \\
\hline 4 & 2 & $-2 \cdot 230-2 \cdot 250$ & - & - & \\
\hline 5 & 5 & - & $-0.763 \quad-0.810$ & - & \\
\hline 6 & $\mathbf{5}$ & - & $-0.723 \quad-0.828$ & - & \\
\hline 7 & 6 & - & 一 & $-0 \cdot 255$ & -0.300 \\
\hline 8 & 6 & $-2 \cdot 075 \quad-2 \cdot 340$ & - & - & \\
\hline 9 & 136 & - & - & $-0 \cdot 288$ & -0.358 \\
\hline 10 & 2 & $-2 \cdot 350$ & $-0 \cdot 673$ & - & \\
\hline 11 & 1 & - & -0.923 & - & \\
\hline 12 & 9 & $-2 \cdot 225^{*}$ & - & - & \\
\hline 13 & 2 & - & - & $-0 \cdot 268$ & -0.358 \\
\hline
\end{tabular}


The variability of the growth rates of type III strains was investigated by determining the differential growth rate, $g_{I}$, of twenty strains each derived from a separate clone. Strains were subcultured from the stock slopes into tubes of broth and the differential growth rates measured simultaneously. For comparison, fifteen well separated colonies of the parent sensitive strain were inoculated into tubes of broth. A mixture was made of each of these cultures with the same type III resistant strain whose growth rate was known, and the rate at which it was eliminated from the mixture of each of the sensitive strains was measured. Assuming a value of $g_{s}$ of 0.845 for the sensitive strain (sce Table 1) it was possible to calculate a series of values of $g_{r}$ for the twenty resistant strains and of $g_{s}$ for the fifteen sensitive strains from equation (1).

\section{Table 4. Comparison of growth rates of twenty type III resistant strains and fifteen sensitive strains}

Twenty type III strains grown with the sensitive strain

$g_{s}=0 \cdot 845$ (assumed). $g_{I}$ determined for each strain $x_{1}=3 \cdot 000$

From equation (1)

$$
g_{r}=0 \cdot 845\left(\frac{3+g_{I}}{3}\right)
$$

Average of values of $g_{r}=0 \cdot 7209$.

Standard deviation of $g_{r}=0 \cdot 0675$.

Fifteen sensitive strains grown with a single type III resistant strain

$g_{I}$ for the resistant strain $=-0.5942$.

Assuming $g_{s}=0 \cdot 845$, then from equation (1) $g_{r}$ for this strain $=2 \cdot 0329$. $x_{1}=3 \cdot 000$.

For the fifteen sensitive strains from equation (1)

Average of values of $g_{s}=\mathbf{0} \cdot 8286$.

$$
g_{s}=\frac{2 \cdot 0329 \times 3}{3+g_{I}}
$$

Standard deviation of $g_{s}=0 \cdot 0437$.

The results are given in Table 4. The standard deviations of the two series were compared using variance of variance $=2 \sigma^{2} /(n-1)$, where $\sigma$ is the variance. For this comparison $t=1 \cdot 61$ and $P=0 \cdot 2-0 \cdot 1$. One can therefore conclude that type III strains, arising independently, show the same degree of variability of their growth rates as would strains derived from individual bacteria of the sensitive population.

Although resistant strains of one type, or occasionally two types, were obtained from $1 \mathrm{ml}$. broth cultures, the sample of the $50 \mathrm{ml}$. cultures in Exp. 1 (Table 1) yielded strains belonging to types I-III and the sample of the $50 \mathrm{ml}$. culture in Exp. 2 (Table 1) yielded types I and III. Furthermore, in both cases the type III strains could be divided into two groups each with a characteristic growth rate. These facts could be explained on the assumption that each clone was derived from a single mutant cell previous to the application of streptomycin. The chances of more than one mutant in $1 \mathrm{ml}$. volumes of culture would be slight, but would be 50 times higher in the $50 \mathrm{ml}$. cultures.

All the type III strains obtained in these four experiments grew at a slower 
rate than the sensitive strain. However, it was possible, by selecting from a large volume of broth culture of the sensitive strain, to obtain a resistant strain which grew as rapidly as its parent.

Table 5. The diameter of colonies of the sensitive and various resistant strains compared with their growth rates by the indirect method

\begin{tabular}{|c|c|c|c|c|c|}
\hline Strain & $g_{I}$ & $\begin{array}{l}\text { Average colony } \\
\text { diameter ( } \mathrm{mm} .)\end{array}$ & $\begin{array}{l}\text { Standard } \\
\text { error }\end{array}$ & $t$ & $\boldsymbol{P}$ \\
\hline Type I & $-2 \cdot 275$ & 0.526 & & & \\
\hline Type I & $-2 \cdot 080$ & $0 \cdot 605$ & & $\{1 \cdot 68$ & $0 \cdot 1$ \\
\hline Type II & $-1 \cdot 540$ & 0.898 & & & \\
\hline Type II & -1.485 & 0.838 & & & $0 \cdot 3-0 \cdot 2$ \\
\hline Type III $b$ & $-0 \cdot 255$ & $2 \cdot 011$ & 0.0331 & & \\
\hline Type III $a$ & -0.923 & $1 \cdot 514$ & & & \\
\hline Type III $a$ & $-1 \cdot 390$ & $1 \cdot 183$ & & & \\
\hline Sensitive & - & 1.940 & & & \\
\hline Adapted 1 & $-1 \cdot 765$ & $0 \cdot 395$ & & $\{2 \cdot 61$ & $0.02-0.01$ \\
\hline Adapted 2 & -0.995 & 0.518 & & & \\
\hline
\end{tabular}

The standard error of the average colony diameter was obtained from the intra-block error term of the analysis of variance.

\section{Colony sizes}

The colony sizes of two type I strains, two type II strains, two type III $a$ strains, one type III $b$ strain, the two adapted strains and the sensitive strain were measured simultaneously. A suitable dilution of each strain was spread on six quarters of nutrient agar plates without streptomycin. Differences between plates were eliminated by the use of the balanced incomplete blocks arrangement (Fisher \& Yates, 1948). After incubation for $14 \mathrm{hr}$. at $30^{\circ}$ and $8 \mathrm{hr}$. at $37^{\circ}$ thirty well separated colonies of each strain were measured. The results are given in Table 5. No difference was found between colony size of the two type I or the type II strains, but the difference between colony sizes of the two adapted strains was significant. There was a reasonable correlation between growth rates and colony size.

\section{Streptomycin resistance}

Type I strains grew in the presence of $6400 \mu \mathrm{g} . / \mathrm{ml}$., the highest concentration used. The number of colonies on these plates was not significantly different from the number on control plates without streptomycin. However, as the concentration of streptomycin in the plates increased growth became progressively slower, and at 3200 and $6400 \mu \mathrm{g} . / \mathrm{ml}$. colonies were often not visible till they had been incubated for $48 \mathrm{hr}$. at $37^{\circ}$. All the strains were identical in their behaviour.

Type II strains also behaved identically. All strains were inhibited by c. $400 \mu \mathrm{g} . / \mathrm{ml}$. There was no significant difference between the numbers of colonies growing on medium containing no streptomycin or $100 \mu \mathrm{g} . / \mathrm{ml}$., and the colony size was only slightly smaller in streptomycin plates. The number of colonies growing on plates containing $200 \mu \mathrm{g}$. $/ \mathrm{ml}$. was less than on control plates and they were small and irregular in size. 
Rather unexpectedly type III strains were found to behave in two quite distinct ways. Type III $a$ strains grew well in the presence of $6400 \mu \mathrm{g} . / \mathrm{ml}$. and the colonies were almost as large as on plates without streptomycin. There was no difference in the number of colonies on plates with or without streptomycin. Type III $b$ strains grew well in $800 \mu \mathrm{g}$. $/ \mathrm{ml}$., but in $1600-6400 \mu \mathrm{g} . / \mathrm{ml}$. growth was very slow and the colonies only became visible after $48 \mathrm{hr}$. incubation at $37^{\circ}$. This slowing of growth, occurring suddenly in concentrations over $800 \mu \mathrm{g} . / \mathrm{ml}$., was characteristic of all these strains and clearly distinguished them from type III $a$ strains. The number of colonies that developed at all streptomycin concentrations did not differ from the number on streptomycin free plates. Strains in Exp. 1 were not tested for resistance in a manner that would distinguish between types III $a$ and III $b$, but in Exps. 2-4 (Tables 3 and 4) it can be seen that all the type III strains in each clone were either III $a$ or III $b$.

\section{Type I strains}

\section{Stability on subculture in broth}

Daily subcultures of eight strains from four separate clones were carried out in broth. Each day an inoculum containing $c$. 100 organisms was spread on plates with and without streptomycin $100 \mu \mathrm{g} . / \mathrm{ml}$. Large colonies began to appear on plates without streptomycin after one or two subcultures, and within 1-3 days they had replaced the original small colonies. Organisms from these large colonies were inhibited by a streptomycin concentration 2-4 times greater than that which would inhibit the sensitive strain. Two of the colonies were inoculated into tubes of broth and were then subcultured daily 31 times. At the end of this period the colonial morphology and streptomycin resistance were unchanged. The growth rate, by the direct method, of one of these strains was 0.632 , only slightly less than 0.845 for the sensitive strain. The distinction between the original type I colonies and the large colonied variants derived from them was well marked and there were no intermediate forms.

Table 6. The average diameter of ten colonies from resistant strains grown on streptomycin free agar before and after forty-two successive daily subcultures in broth

\begin{tabular}{|c|c|c|}
\hline \multirow[b]{2}{*}{ Strain } & \multicolumn{2}{|c|}{$\begin{array}{l}\text { Average diameter of colonies and its } \\
\text { standard error }\end{array}$} \\
\hline & $\begin{array}{l}\text { Before subculture } \\
\text { (mm.) }\end{array}$ & $\begin{array}{l}\text { After subculture } \\
\text { (mm.) }\end{array}$ \\
\hline Type II & $0.90 \pm 0.014$ & $1 \cdot 73 \pm 0 \cdot 044$ \\
\hline Type III $a$ & $1 \cdot 05 \pm 0.025$ & $1 \cdot 46 \pm 0.034$ \\
\hline Type III $b$ & $1.56 \pm 0.034$ & $1 \cdot 70 \pm 0 \cdot 044$ \\
\hline Sensitive & \multicolumn{2}{|c|}{$1.80 \pm 0.048$} \\
\hline
\end{tabular}

\section{Type II strains}

These strains were also unstable though in a less clear-cut fashion. There was a slight fall in resistance occurring after 5-16 daily subcultures. The strains were now inhibited by a streptomycin concentration of $100 \mu \mathrm{g} \cdot / \mathrm{ml}$. 
At the same time larger colonies of two sizes began to appear on streptomycin free plates. Usually, though not invariably, the larger colonies were less resistant than the small ones. After a total period of subculture of 2-6 weeks a further fall in streptomycin resistance occurred. The strains now contained a majority of organisms only 4-8 times less sensitive than the sensitive strain, but there was also present a small proportion of the more resistant organisms inhibited by $100 \mu \mathrm{g} . / \mathrm{ml}$. During further subculture this proportion varied from 1 to $50 \%$ of the total culture, but showed no signs of being eliminated. By this time all colonies on streptomycin free plates were of fairly uniform size and as large as the parent strain. Measurements of colony size are given in Table 6. These results appear to indicate that the fall in resistance occurred in two stages, although complete reversion to the original level of sensitivity of the parent strain did not occur.

\section{Type III strains}

A group of three type III $a$ strains with widely different growth rates and a type III $b$ strain were still able to grow in the presence of $6400 \mu \mathrm{g} . / \mathrm{ml}$. streptomycin after forty-two daily subcultures in broth. The type III $b$ strain also showed the same slow growth in streptomycin concentrations of 1600 $6400 \mu \mathrm{g} . / \mathrm{ml}$. as it did originally.

Type III strains with a slow growth rate and a small colony size, when subcultured in broth and plated out at intervals on plates without streptomycin, produced after a few days a number of colony variants which were larger than the original colonies. After about a month's subculture, as is shown in Table 6, the colonies were almost as large as those of the sensitive strain. This phenomenon was investigated in greater detail in an experiment in which two clones of type III resistant strains, one with growth rates almost as rapid as the sensitive strain and the other with slow growth rates, were mixed with the sensitive strain and the fall in the numbers of resistant organisms determined. This was carried out immediately after isolation and after subculture of the resistant strains for 7 and 21 days in streptomycin free broth.

The slowly growing strains increased their growth rates after subculture in plain broth, but to differing extents. Also, during the periods of the tests done after 7 and 21 days' subculture, the rate for any one strain was more variable. In particular, the behaviour of one strain in the mixture made after $r$ days was notable in that for 8 days it had a slow growth rate; then it grew as well as the sensitive strain for 5 days and finally the growth rate again became slow. Then again, one of the two strains from the clone that grew rapidly maintained its growth rate in the mixture made after 7 days, but the other strain, after maintaining its growth rate for 6 days, began to grow increasingly slowly. In the mixture made after 21 days the same phenomenon occurred but the behaviour of the strains was reversed.

One could conclude that the constancy of the growth rate characteristic of type III resistant strains belonging to a single clone was lost after 21 days' subculture in streptomycin free medium and was replaced by a variability similar in degree to that found between strains derived from a number of 
different clones. Although most strains increased their growth rates a few spontaneously decreased their growth rates for shorter or longer periods.

Type III $a$ strains tested shortly after isolation produced colonies of about the same size whatever the concentration of streptomycin in the plates. However, after forty-two daily subcultures in broth, the three strains tested yielded colonies that were smaller with progressively increasing streptomycin concentrations, so that on $400 \mu \mathrm{g} . / \mathrm{ml}$. or above the colonies were smaller than those produced by the original strain.

Growth rates of resistant strains mixed in varied proportions with the sensitive strain. It might be thought that the behaviour of mixtures of equal quantities of sensitive and resistant organisms would not reflect the population dynamics in other cases where the proportions differed widely. When two type III strains were mixed in widely different proportions with the sensitive strain their growth rates were found to be the same whatever the original proportion of the resistant to sensitive organisms. The proportions of resistant to sensitive organisms in this experiment were $1: 10^{3}, 1: 1,10^{3}: 1$ and $10^{6}: 1$ (in the last case the inoculum contained about fifty sensitive organisms). In the cases where the resistant organisms predominated the differential growth rates were calculated by an extension of the method used for obtaining equation (1).

\section{Adapted strains}

The results of testing the differential growth rate and the streptomycin sensitivity of the two adapted strains are shown in Table 7. The average colony sizes on nutrient agar, compared with those of other resistant types, are

Table 7. Comparison of the growth rates and streptomycin sensitivities of the adapted strains with type I and type II strains

$\overbrace{\text { Type }}^{\text {Strain }}$

Differential
growth rate
$\left(g_{I}\right)$
$-1 \cdot 765$
-0.995
$-2 \cdot 080$
$-2 \cdot 275$
$-1 \cdot 485$
$-1 \cdot 540$

Minimal inhibitory
concentration of
streptomycin
$(\mu \mathrm{g} . / \mathrm{ml}$.
1600
1600
$>6400$
$>6400$
400
400

shown in Table 5. The two strains had different growth rates, both being rather slow and corresponding most closely to those of type II resistant strains. They were both partially resistant to streptomycin, being inhibited by $1600 \mu \mathrm{g} . / \mathrm{ml}$. After 2 days' subculture in broth without streptomycin there was no change in streptomycin resistance. After 3 days' subculture, however, large colonied variants began to appear which were less resistant to streptomycin. After 7 days' subculture both strains were mainly composed of organisms inhibited by $8-32 \mu \mathrm{g} . / \mathrm{ml}$., together with about $10 \%$ of organisms inhibited by $200 \mu \mathrm{g} . / \mathrm{ml}$. Adapted strains corresponded in their behaviour on 
subculture to type II strains except that the changes occurred more rapidly. They also correspond in streptomycin sensitivity and growth rates most closely to type II strains, although in each case there were definite differences in behaviour.

\section{Mutation rate}

The mutation rates to resistance of types I-III, to resistance of all types and to dependence were calculated using Newcombe's (1948) formula (1):

$$
\text { Mutation rate }=-\frac{\left(\log _{e} 2\right)\left(\log _{e} P_{0}\right)}{N}
$$

where $\boldsymbol{P}_{\mathbf{0}}=$ proportion of cultures without mutants and $N=$ number of bacteria per culture. This estimation is unaffected by the relative growth rates of sensitive and resistant strains.

The results from Exps. 1-3 are given in Table 8. Separate rates for types III $a$ and III $b$ could not be calculated because the data were insufficient. However, out of nineteen clones tested in Exps. 2-4, fifteen were type III $a$ and four type III $b$. The mutation rates to the different resistant types do not differ significantly from each other.

Table 8. Mutation rates for resistant and dependent strains

\begin{tabular}{|c|c|c|c|c|}
\hline & \multicolumn{3}{|c|}{ Experiment } & \multirow[b]{3}{*}{ Average } \\
\hline & 1 & 2 & 3 & \\
\hline & \multicolumn{3}{|c|}{ Mutation rate $\times 10^{-11}$} & \\
\hline All resistant types & $8 \cdot 6$ & $14 \cdot 5$ & $11 \cdot 7$ & $11 \cdot 6$ \\
\hline Resistant type I & $2 \cdot 2$ & $5 \cdot 8$ & $7 \cdot 0$ & $5 \cdot 0$ \\
\hline Resistant type II & $0 \cdot 87$ & $2 \cdot 2$ & $1 \cdot 4$ & $1 \cdot 5$ \\
\hline Resistant type III & 4.7 & $9 \cdot 8$ & $4 \cdot 0$ & $6 \cdot 2$ \\
\hline Dependent & $21 \cdot 3$ & $18 \cdot 1$ & $20 \cdot 5$ & $20 \cdot 0$ \\
\hline
\end{tabular}

\section{DISCUSSION}

These experiments show that streptomycin-resistant strains obtained from a sensitive population by single step selection could be divided into three main types. Type I strains had a slow growth rate, were completely resistant, though growing poorly at high streptomycin concentrations, and were markedly unstable when subcultured in broth. Type II strains had a slightly more rapid growth rate, were partially resistant and were unstable when subcultured in broth, though much less so than type I strains. Type III strains had a variable growth rate, usually more rapid than type I or type II strains, were completely resistant and were stable on subculture. Type III strains could be subdivided on the basis of only one character. Type III $a$ strains grew almost as well in the absence as in the presence of high concentrations of streptomycin, whereas type III $b$ strains grew much more slowly on plates containing $1600 \mu \mathrm{g} . / \mathrm{ml}$. or over. These characters were all quite clearly defined and in no case was there any doubt as to which type any strain should be assigned.

Evidence for the existence of streptomycin-resistant mutants in a sensitive population before the selective contact with the drug has been presented by 
Alexander \& Leidy (1947), Demerec (1948), Meads \& Haslam (1949), Scott (1949), English \& McCoy (1951) and Lederberg \& Lederberg (1952).

These results provide further evidence for this view, since the only reasonable explanation for the occurrence of clones of resistant colonies having identical characters is that they were each derived from a single mutant cell which had the opportunity to divide a few times before contact with streptomycin.

The $1 \mathrm{ml}$. broth cultures of the sensitive strain were inoculated with a number of organisms too small to contain any resistant mutants. Since strains of each of the types described were obtained from a number of these independent cultures it is reasonable to consider that each type arose by a separate mutation. If one assumes that mutation rate to types I-III resistance are the same, one can calculate from the data in Tables 2,3 and 8 how many $1 \mathrm{ml}$. cultures in Exps. 1-3 would be likely to contain mutants of two different types. These calculated values are $0.1,0.9$ and 0.6 , whereas in these experiments respectively 0,4 and 1 cultures actually contained mutants of two types. Using the same data one can calculate that the most likely number of resistant clones to be obtained from the $50 \mathrm{ml}$. volumes of the sensitive strain in Exps. 1 and 2 would have been about 3 and 9 . In fact, the numbers found were 4 and 3. It is therefore reasonable to assume that the mutations arose independently of each other.

None of the resistant strains which was subcultured in streptomycin free broth reverted to the sensitivity of the sensitive strain. All type I strains and the large colonied variant strains derived from them had slower growth rates than the sensitive strain. Type II strains had slower growth rates for at least the first fortnight of daily subcultures. Type III strains usually had slower growth rates, and even where these were as rapid as the sensitive strain the data in Fig. 4 indicate that they tended to revert permanently or temporarily to a slower rate. A mixed population, containing initially only fifty sensitive organisms and a much larger number of a resistant strain with a slower growth rate, was shown after 3 days' subculture to contain a majority of sensitive organisms. It may be concluded, therefore, that back mutation to a strain identical with the sensitive strain does not occur or is extremely rare. This assumes that the back mutant, if it occurred at all, would appear sufficiently early in the growth in one tube for its progeny to have a chance of being transmitted in the inoculum to the next tube. Such a condition is likely to have been fulfilled in one of the large number of tubes in which the resistant strains were subcultured.

In two experiments with large volumes of broth cultures of the sensitive strain the proportion of resistant and dependent organisms to sensitive organisms was found to be $1: 5.8 \times 10^{8}$ and $1: 8 \cdot 2 \times 10^{8}$. The maintenance of this ratio between a large number of sensitive organisms and a small number of resistant ones can be explained as a balance between two tendencies. The population of resistant organisms is constantly increased by new mutants. If the resulting resistant strains grew as well as the sensitive strain they would eventually replace it, since it appears that back mutation either does not occur 
or occurs very rarely. However, this tendency for the resistant population to increase is counterbalanced by the slower growth rate of resistant strains and the failure of dependent strains to grow for more than two divisions (Newcombe \& Hawirko, 1949). As an example in which this does not occur English \& McCoy (1951) found that streptomycin-resistant variants of Staphylococcus aureus grew better than the sensitive strain in a semi-synthetic medium and were able to obtain a streptomycin-resistant strain by inoculating a large number of tubes of this medium with sensitive organisms.

Speculations about the nature of resistance to streptomycin tend to emphasize how little is known about it. Two comments can, however, be based on these results. The variability of sensitive strains derived from separate colonies suggests that individual bacilli in a sensitive population have characteristic growth rates which are transmissible to daughter cells during several periods of daily subculture. The variability in these growth rates was also found in type III resistant strains and is most likely to be due to several factors. Their effect has, however, been completely suppressed in types I and II strains, whose growth rates appeared identical. An investigation into the causes of variability among sensitive and type III resistant strains might provide evidence as to the nature of the mechanism of types I and II resistance.

The second comment springs from consideration of the colony sizes of resistant strains in the absence and in the presence of streptomycin. Oginsky, Smith \& Umbreit (1949) have suggested that streptomycin acts by preventing an oxaloacetate-pyruvate condensation and that this condensation does not occur in resistant strains. From this hypothesis one would expect the growth rate of resistant strains to be unaffected by streptomycin. However, type I colonies were found to be very much smaller in the presence of high streptomycin concentration than in its absence. Type II strains were inhibited at concentrations over $400 \mu \mathrm{g}$. $/ \mathrm{ml}$. Type III $b$ colonies were small in the presence of $1600 \mu \mathrm{g} . / \mathrm{ml}$. or more. Type III $a$ colonies, although initially of equal size in the presence or absence of streptomycin, were much smaller in its presence after a period of subculture in broth. Any hypothesis, such as this, that explained the action of streptomycin by an effect on a site which is present in sensitive strains but completely absent in resistant strains is thus unlikely to provide a full explanation.

Newcombe \& Nyholm (1950) have provided evidence from experiments with sexual matings of Bact. coli, strain K-12, that streptomycin resistance and dependence arise through mutation in the same single gene locus. It would appear likely that their resistant strains were type III since, under the conditions they describe for obtaining their strains, type II colonies would not appear and type I colonies would be very small. Whether types III $a$ and III $b$ strains arise from the same or a different locus is undetermined.

Type II strains resemble the adapted strains most closely. There is evidence that several mutational steps are involved in the production of adapted strains (Demerec, 1948), and that some of these involve the locus for resistance and dependence (Newcombe \& McGregor, 1951). The stepwise fall in resistance of 
type II strains when subcultured in the absence of streptomycin suggests that multiple mutations are also involved in the production of these strains. Since they were obtained by a single step selection, these mutations must have been present simultaneously in the same organism and each must therefore be relatively frequent in occurrence.

Type I strains subcultured in the absence of streptomycin produced stable variant strains with a higher growth rate and lower resistance. This would suggest either that two mutations are involved in the production of these strains, from one of which there are frequent back mutations, or that the variant strains result from the effect of a blocking gene on an original single mutation.

The genetic mechanisms need further investigation, and the occurrence of these types of streptomycin resistance must be confirmed in other strains of Bact. coli before generalizations can be made.

This work was carried out during the receipt of a grant from the Medical Research Council, and with the technical assistance of Mr D. J. Rose. I am indebted to Dr W. Hayes for his interest and criticisms.

\section{REFERENCES}

Alexander, H. E. \& Leidy, G. (1947). Mode of action of streptomycin on type $b$ H. influenzae. I. Origin of resistant organisms. J. exp. Med. 85, 329.

Demerec, M. (1948). Origin of bacterial resistance to antibiotics. J. Bact. 56, 63. ENGLISH, A. R. \& MCCoy, M. (1951). Growth comparisons of streptomycin-sensitive and streptomycin-resistant Micrococcus pyogenes var. aureus. J. Bact. 62, 19.

Fisher, R. A. \& Yates, F. (1948). Statistical Tables for Biological, Agricultural and Medical Research, 3rd ed. p. 19. London: Oliver and Boyd.

Lederberg, J. \& LeDerberg, E. M. (1952). Replica plating and indirect selection of bacterial mutants. J. Bact. 63, 399 .

Meads, M. \& Haslam, N. M. (1949). Quantitative studies on the origin and characteristics of streptomycin-fast variants of Klebsiella pneumoniae. J. Immunol. $63,1$.

Newcombe, H. B. (1948). Delayed phenotypic expression of spontaneous mutations in Escherichia coli. Genetics, 33, 447.

Newcombe, H. B. \& Hawirko, R. (1949). Spontaneous mutation to streptomycin resistance and dependence in Escherichia coli. J. Bact. 57, 565.

Newcombe, H. B. \& Nyноцm, H. H. (1950). The inheritance of streptomycin resistance and dependence in crosses of Escherichia coli. Genetics, 35, 603.

Newcombe, H. B. \& McGregor, J. (1951). On the non-adaptive nature of change to full streptomycin resistance in Escherichia coli. J. Bact. 62, 539.

Oginsky, E. L., Smith, P. H. \& Umbreit, W. W. (1949). The action of streptomycin. I. The nature of the reaction inhibited. J. Bact. 58, 747 .

ScotT, G. W. (1949). Spontaneous mutation to streptomycin resistance in Escherichia coli. Brit. J. exp. Path. 30, 501. 